\title{
Influências do ciclo estral sobre o desempenho de ratos no labirinto em cruz elevado
}

\author{
Amauri Gouveia Júnior \\ Universidade Estadual Paulista Júlio de Mesquita Filho \\ Silvio Morato \\ Faculdade de Filosofia, Ciências e Letras de Ribeirão Preto
}

\begin{abstract}
Resumo
O Labirinto em cruz elevado é um dos mais populares modelos animais para estudo da ansiedade. Esta pode ser influenciada por fatores fisiológicos como o ciclo hormonal das fêmeas. O objetivo do presente trabalho foi investigar as influências do ciclo estral no comportamento exploratório de ratas no Labirinto em cruz elevado. Setenta animais (13 machos e 61 fêmeas) foram utilizados. As fêmeas tiveram seu ciclo estral mapeado e os animais foram testados segundo a fase do ciclo em que se encontravam, gerando, além do grupo Machos, 3 grupos de fêmeas: Proestro $(\mathrm{N}=23)$, Estro $(\mathrm{N}=19)$ e Diestro $(\mathrm{N}=19)$. Cada animal foi submetido a uma única sessão de 5 min. no Labirinto. Os resultados indicam diferenças significativas entre o grupo de machos e o de fêmeas no Diestro quanto à porcentagem de tempo gasto nos braços abertos, mas não quanto à percentagem de entradas nos mesmo.
\end{abstract}

Palavras-chave: ciclo estral; ansiedade; labirinto em cruz elevado.

\begin{abstract}
Effects of Estrous cycle on the behavior in the elevated plus maze

The Elevated plus maze is one of the most popular models to study animal anxiety. Anxiety can be influenced by physiological factors, such as the hormonal state. The aim of the present work is to investigate the relationship between estrus cycle and rat exploratory behavior in the elevated plus maze. The study made use of male $(n=13)$ and female rats $(n=61)$. The females had their estral phase identified and were divided into 3 groups according to their hormonal state: Proestrus $(\mathrm{N}=23)$, Estrous $(\mathrm{N}=19)$ and Diestrous $(\mathrm{N}=19)$. All animals were submitted to a 5-min session in the elevated plus maze. The data indicate differences between the diestrus group and male group in the percentage of time spent in the open arms, but do not in the percentage of entries.
\end{abstract}

Keywords: estral cycle; anxiety; elevated plus maze.

O Labirinto em cruz elevado é um modelo animal de ansiedade desenvolvido na década de 80 (Handley \& Mithani, 1984; Pellow, Chopin, File \& Briley, 1985; Pellow \& File, 1986) com base em experimentos feitos na década de 50 com uma versão modificada do Labirinto em Y, que possuía um dos braços aberto e elevado do chão (Montgomery, 1955). O Labirinto em cruz elevado é composto por um $\mathrm{X}$ de madeira elevado a $50 \mathrm{~cm}$ do chão e dotado de dois braços abertos e dois fechados por paredes e opostos entre si. Sua utilização como medida de ansiedade é baseada na aversão incondicionada do animal por lugares abertos e sua validade como modelo animal de ansiedade é baseada na sua sensibilidade a inúmeros agentes ansiolíticos e ansiogênicos (Hogg, 1996), bem como a tratamentos estressores tais como nado forçado, isolamento e choques, entre outros (Pellow e cols., 1985; Pellow \& File, 1986; Hogg, 1996; Morato \& Brandão, 1997; Cole, Hillman, Seidelmann, Klewer \&
Jones, 1995). O tempo gasto em cada braço, o número de entradas nos braços abertos e as porcentagens de tempo e entradas são seus parâmetros mais usados, sendo a permanência nos braços abertos um índice confiável de ansiedade (Pellow e cols., 1985).

Nas ratas, o ciclo hormonal dura de quatro a cinco dias e tem quatro fases, denominadas de Estro, Metaestro, Diestro e Proestro. O Estro corresponde à ovulação e nesta fase a progesterona encontra-se em seu nível máximo. As células da vagina apresentam-se cornificadas, com intensa descamação. O Metaestro corresponde à fase entre os ciclos, na qual não há ação hormonal identificável e as células vaginais se mostram diversificadas. O Diestro corresponde à ação inicial do estradiol sobre o organismo, com níveis de estradiol correspondentes à metade da taxa máxima, sendo que as células da vagina não apresentam descamação e há grande número de leucócitos. O Proestro corresponde ao pico da ação do estradiol, com as 
células da vagina apresentando-se grandes e nucleadas, sem leucócitos. (Schwatz, 1969; Carter, 1993; Kandel, Schwatz \& Jessell, 1995).

Embora as influências do ciclo estral sobre o desempenho comportamental de ratos sejam conhecidas desde o início do século XX, apenas a partir da década de 70 estudos sistemáticos sobre diferenças de gênero em modelos animais de ansiedade passaram a ser feitos em larga escala (Breedlove, 1993). Uma súmula não exaustiva dos resultados sobre este tema é apresentada na Tabela 1.

Tabela 1 - Exemplos de diferenças de gênero em modelos animais descritas na literatura

\begin{tabular}{|c|c|c|c|c|}
\hline Modelos animais & Parâmetro & $\hat{\sigma}$ & $q$ & Autores \\
\hline \multirow[t]{3}{*}{ Labirinto em Cruz elevado } & Taxa de entradas nos braços abertos & & + & $\begin{array}{l}\text { Johnston e File, 1991; } \\
\text { Steenbergen e cols., } 1990\end{array}$ \\
\hline & Permanência nos braços abertos & & + & Johnston e File, 1991 \\
\hline & $\begin{array}{l}\text { Efeitos de choques elétricos sobre } 0 \\
\text { desempenho }\end{array}$ & + & & Steenbergen e cols., 1990 \\
\hline Esquiva de duas vias & aquisição & & + & Beatty e Beatty, 1970 \\
\hline Interação social & Contactos & + & & Johnston e File, 1991 \\
\hline Beber punido (Teste de Vogel) & Tempo de supressão & + & & Johnston e File,1991 \\
\hline \multirow[t]{2}{*}{ Campo Aberto } & Deslocamento & & + & $\begin{array}{l}\text { Curzon, Haaren e Kennett, 1990; Kennett, } \\
\text { Chauloff, Marcou e Curzon, } 1986\end{array}$ \\
\hline & Defecação & + & & $\begin{array}{l}\text { Alonso, Castellano, Afonso e Rodriguez, 1991; } \\
\text { Curzon e cols., 1990; } \\
\text { Kennett e cols., } 1986\end{array}$ \\
\hline Desespero comportamental & Atividade & & + & $\begin{array}{l}\text { Alonso e cols., 1991; } \\
\text { Kennett e cols., } 1996\end{array}$ \\
\hline Desamparo aprendido & $\begin{array}{l}\text { Efeito no Labirinto em Cruz elevado } \\
\text { Teste de aprendizagem de } \\
\text { esquiva/fuga de choques }\end{array}$ & $\begin{array}{l}+ \\
+\end{array}$ & $\begin{array}{l}+ \\
=\end{array}$ & $\begin{array}{l}\text { Steenbergen e cols, } 1990 \\
\text { Steenbergen e cols., 1990; } \\
\text { Steenbergen e cols., 1989; } \\
\text { Moran e Lewis-Smith, } 1979 \\
\text { Parra, Padilla, Segovia e Guilamón, } 1990 \\
\text { Gouveia Jr, 2001; } \\
\text { Damiani e Hunziker, 1991; Heinsbroek, Van } \\
\text { Haaren, Poll e Steenbergen, } 1991\end{array}$ \\
\hline Restrição & $\begin{array}{l}\text { Exploração do campo aberto } \\
\text { defecação }\end{array}$ & + & + & Curzon e cols., 1990 \\
\hline Agressão defensiva & $\begin{array}{l}\text { Vocalizações Pré, inter e pós-choques } \\
\text { Vocalizações Freqüência e duração }\end{array}$ & + & + & Blanchard, Franelly e Blanchard, 1986 \\
\hline Reatividade ao estresse & Parâmetros fisiológicos de resposta & + & & $\begin{array}{l}\text { Zukowska-Grojec, Shen, Capraro e Vaz, 1991; } \\
\text { Liversey, Balabkins e Vogel, } 1987\end{array}$ \\
\hline Reatividade a forma de alojamento & $\begin{array}{l}\text { Alojamento individual } \\
\text { alojamento grupal }\end{array}$ & + & + & Brown e Grunberg, 1995 \\
\hline
\end{tabular}

Onde: $\widehat{\delta}=$ machos e $q=$ fêmeas

Normalmente, estes estudos listados apresentavam grande dispersão nos grupos de fêmeas. Uma das hipóteses possíveis para explicar estes dados era a influência do ciclo hormonal das fêmeas. Ainda assim, pou- cos estudos objetivaram investigar as diferenças relativas ao ciclo estral em relação aos modelos animais de psicopatologia. Uma súmula não exaustiva destes estudos é apresentada na Tabela 2.

Tabela 2 - Exemplos de diferenças em modelos animais relativas ao ciclo estral relatadas na literatura

\begin{tabular}{|c|c|c|c|c|c|c|}
\hline \multirow{2}{*}{ Modelos animais } & \multirow{2}{*}{ Parâmetro } & \multicolumn{4}{|c|}{ Fases } & \multirow{2}{*}{ Autores } \\
\hline & & $D$ & $M$ & $P$ & $E$ & \\
\hline Labirinto em Cruz elevado & Taxa de entrada nos braços abertos & + & & & & Dia-Veliz e cols., 1989, 1997 \\
\hline Labirinto em T elevado & Esquiva inibitória do braço fechado & + & & & & Gouveia Jr., 2001 \\
\hline Esquiva de duas vias & Aquisição & + & & & & Diaz-Veliz e cols., 1989 \\
\hline \multirow{2}{*}{ Labirinto de Morris } & Tarefa com dica & & & + & & Warren e Juraska, 1997 \\
\hline & Aprendizagem espacial & & & & + & Warren e Juraska, 1997 \\
\hline Analgesia induzida por choque & Índice de analgesia & & & & + & Ryan e Maier, 1988 \\
\hline Ingestão alimentar a longo prazo & Ingestão & & + & + & & Laviano e cols., 1996 \\
\hline
\end{tabular}




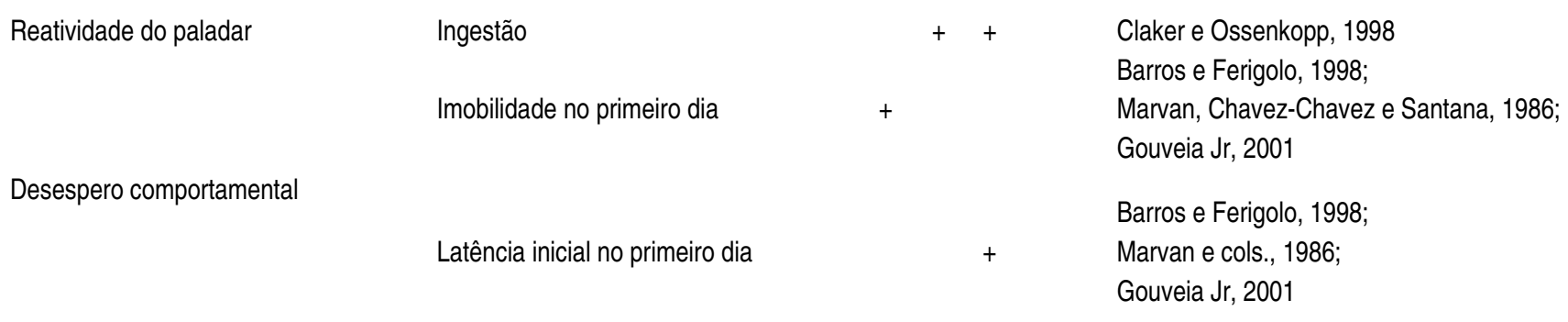

Onde: $\mathrm{D}=$ Diestro; $\mathrm{M}=$ Metaestro; $\mathrm{P}=$ Proestro e $\mathrm{E}=$ Estro

Os dados apresentados na tabela indicam que o ciclo estral é uma variável crítica na aquisição da esquiva condicionada de duas vias, ocorrendo facilitação da aprendizagem da mesma durante o Diestro (DiazVeliz, Soto, Dussaubat \& Mora, 1989). Há também relatos de diferenças entre as fases do ciclo na inibição pré-pulso ${ }^{3}$ (Koch, 1998). No Labirinto aquático de Morris, no Proestro, há um melhor desempenho na tarefa com dicas, com o animal apresentando menor latência para encontrar a plataforma, enquanto, no Estro, há uma melhora na aprendizagem espacial, ou seja, na capacidade de aprendizagem de local (Warren \& Juraska, 1997). O estado hormonal também atenua a analgesia induzida durante o Estro (Ryan \& Maier, 1988), mas parece não alterar a aquisição de comportamento alimentar gerado por estresse crônico (Anderson, Saviolaskis, Bauman, Chu, Ghosh \& Kant, 1996), embora altere a ingestão alimentar a longo prazo (Laviano, Meguid, Gleason, Yang \& Renvyle, 1996). Além disso, a reatividade do paladar também é dependente do ciclo, aumentando no Proestro e no Diestro (Clarke \& Ossenkopp, 1998), e a resposta do beber induzido por insulina, de forma fase-dependente, ou seja, o índice medido (beber) varia não somente com a dose do hormônio (insulina) mas também com a fase do ciclo na qual o sujeito se encontra (Fernandez-Trisac, Brime, López-Sela, Sanches-Garrido, Costales, Argüelles \& Vijande, 1998).

O comportamento exploratório no Labirinto em cruz elevado é alterado por várias condições hormonais, como lactação e a pinealectomia (Silva, Bernadi, Nasello \& Felicio, 1997; Padovan, Del-Bel \& Guimarães, 1996; Kellogg \& Barrett, 1999). Existem estudos relativos às diferenças sexuais (Johnston \& File, 1991; Steenbergen, Heinsbroek, Van Hesta \& Poll, 1990) demonstrando que fêmeas apresentam maior quantidade e tempo de exploração dos braços abertos; tais estudos, no entanto, não manipularam o ciclo estral.
Este foi objeto de dois experimentos, que relatam a manipulação do ciclo hormonal em testes no Labirinto em cruz elevado. Um desses trabalhos manipulou o ciclo estral e obteve aumentos significativos tanto na porcentagem de entradas nos braços abertos durante $o$ Diestro, mas somente em condições de baixa luminosidade, quanto na porcentagem de tempo do grupo Metaestro, na condição de alta luminosidade (Mora, Dussaubat \& Diaz-Veliz, 1996). Neste trabalho não foram comparados os gêneros. No mesmo artigo, em outro experimento, ratas castradas foram submetidas a injeções agudas de benzoato de estradiol ou progesterona e encontraram-se diferenças entre os grupos que receberam progesterona e aqueles que receberam somente veículo ou estradiol, ou, ainda, que receberam ambos os hormônios combinados (Mora e cols., 1996). Em ambos os experimentos, houve diferenças entre alta luz e baixa luz (Mora e cols., 1996), muito embora Becker \& Grecksch (1996) relatem que esta não é uma variável crítica, pelo menos para os machos.

Em outro trabalho do mesmo grupo (Diaz-Veliz, Alarcon, Espinoza, Dussaubat \& Mora, 1997), são relatadas diferenças significativas entre os grupos, com maiores porcentagens de entrada e tempo dispendido nos braços abertos no Proestro e no Estro em relação aos demais grupos (incluindo, neste trabalho, um de Machos).

Recentemente outro trabalho (Reddy \& Kulkarni, 1999) apresentou dados relativos ao ciclo estral medidos no Labirinto em cruz elevado utilizando este teste como medida de memória, por meio da análise da latência de transferência entre os braços fechados e abertos. Infelizmente, esses dados não são comparáveis, pois a manipulação dos dados usada foi diametralmente diversa daquela dos trabalhos citados até aqui. 
O objetivo do presente trabalho foi o de replicar, de forma parcial, o trabalho de Mora e cols. (1996). A replicação caracteriza-se como parcial pelo que segue: 1) apenas um dos experimentos citados no artigo original de Mora será refeito; 2) as fases Metaestro e Diestro serão consideradas como uma única fase; 3 ) os parâmetros do aparato estão de acordo com a maior central da Unesp (Botucatu, SP), experimentalmente ingênuos, com aproximadamente 100 dias de vida no início dos experimentos e pesando cerca de 210 g. Os animais foram mantidos em gaiolas coletivas $(n=5)$ com comida e água ad lib e em um ciclo claro/escuro de 12 horas, com as luzes acesas às 7 horas da manhã.

Equipamento. Foi utilizado um Labirinto em cruz

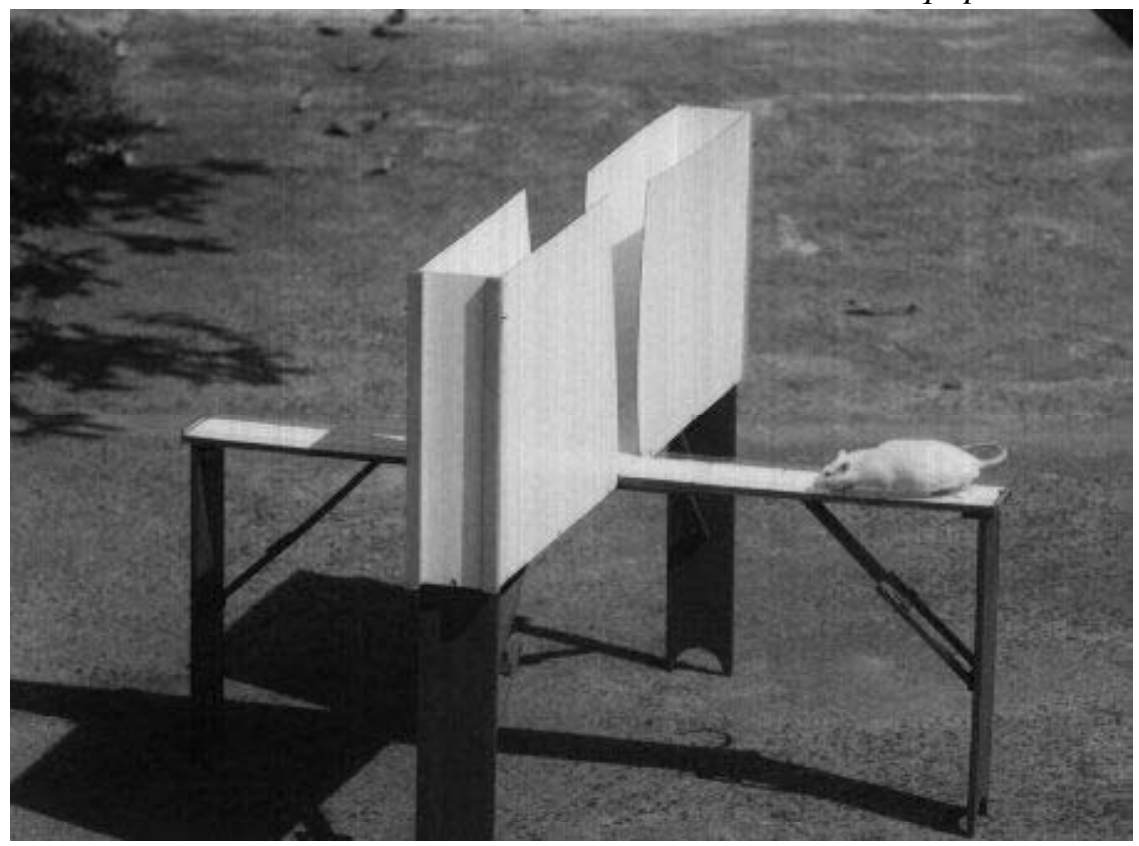

parte da literatura da área, com altura de $50 \mathrm{~cm}$ do chão (contra $70 \mathrm{~cm}$ utilizado por Mora e cols., (1996) e paredes de $40 \mathrm{~cm}$ nos braços fechados, contra $20 \mathrm{~cm}$ utilizados no trabalho citado).

\section{MÉTODO}

Sujeitos. Foram usados setenta e quatro ratos Wis$\operatorname{tar}$ (13 machos e 61 fêmeas) provenientes do biotério

Procedimento. As fêmeas foram mapeadas em seu ciclo estral por coleta de esfregaço vaginal diário por um período mínimo de cinco dias antes dos testes e distribuídas em grupos conforme a fase do ciclo no primeiro dia do experimento, resultando assim em 4 grupos: Estro $(\mathrm{N}=19)$, Diestro $(\mathrm{N}=19)$, Proestro ( $\mathrm{N}$ $=23)$ e Machos $(\mathrm{N}=13)$. Os machos não foram submetidos a nenhuma manipulação anterior. Todos os animais foram pesados no dia do teste, após a sessão experimental. Todos os experimentos e coletas de esfregaços foram realizados entre as $9 \mathrm{~h}$. e $14 \mathrm{~h}$. e foram filmados, sendo os dados coletados transcritos, usando-se o software Etholog 2.0 (Othoni, 2000) e posteriormente tabulados. Cada sujeito foi submetido a uma única sessão de 5 minutos no Labirinto. A sessão tinha início quando o animal era colocado "gentilmente" no centro do Labirinto, com o focinho voltado para um dos braços fechados. Foram registrados as entradas e o tempo gasto em cada braço. No inter- elevado, consistindo de dois braços abertos $(50 \times 10$ $\mathrm{cm})$ e dois fechados (50 x $10 \times 40 \mathrm{~cm})$, arranjados de forma que os braços de mesmo tipo fiquem em oposição entre si, ligados por uma área central $(10$ x $10 \mathrm{~cm})$. $\mathrm{O}$ aparato todo ficava a $50 \mathrm{~cm}$ do chão. O Labirinto era todo feito de madeira e o piso dos braços era revestido de fórmica branca. A Figura 1 apresenta foto do aparato usado.

valo entre as sessões, o aparato era limpo com uma solução de $10 \%$ de etanol. Foram observados a frequiência de entradas, o tempo de permanência e a porcentagem (taxa) de entradas e de permanência dos sujeitos em cada um dos tipos de braço do aparato.

Análise estatística. A análise estatística foi feita usando-se análise de variância (ANOVA) de duas vias que faz parte do pacote estatístico Sistat 3.0 para computadores, considerando-se o peso corporal como covariante. Sempre que apropriado, as ANOVAs eram seguidas pelo teste de Tukey, de comparações entre médias de grupos. Em todos os casos adotou-se um alfa de 0,05 como significativo.

\section{RESULTADOS E DISCUSSÃO}

A Figura 2 apresFigura 1. O Labirinto em cruz elevadoas e do tempo de perm(Foto: Prof. Titular K. Hoshino) os da porcentagem de cnuauas c uc ınıрu gasu nos braços para os diversos grupos. 
— Machos $\square$ proestro $\square$ Estro $\square$ diestro
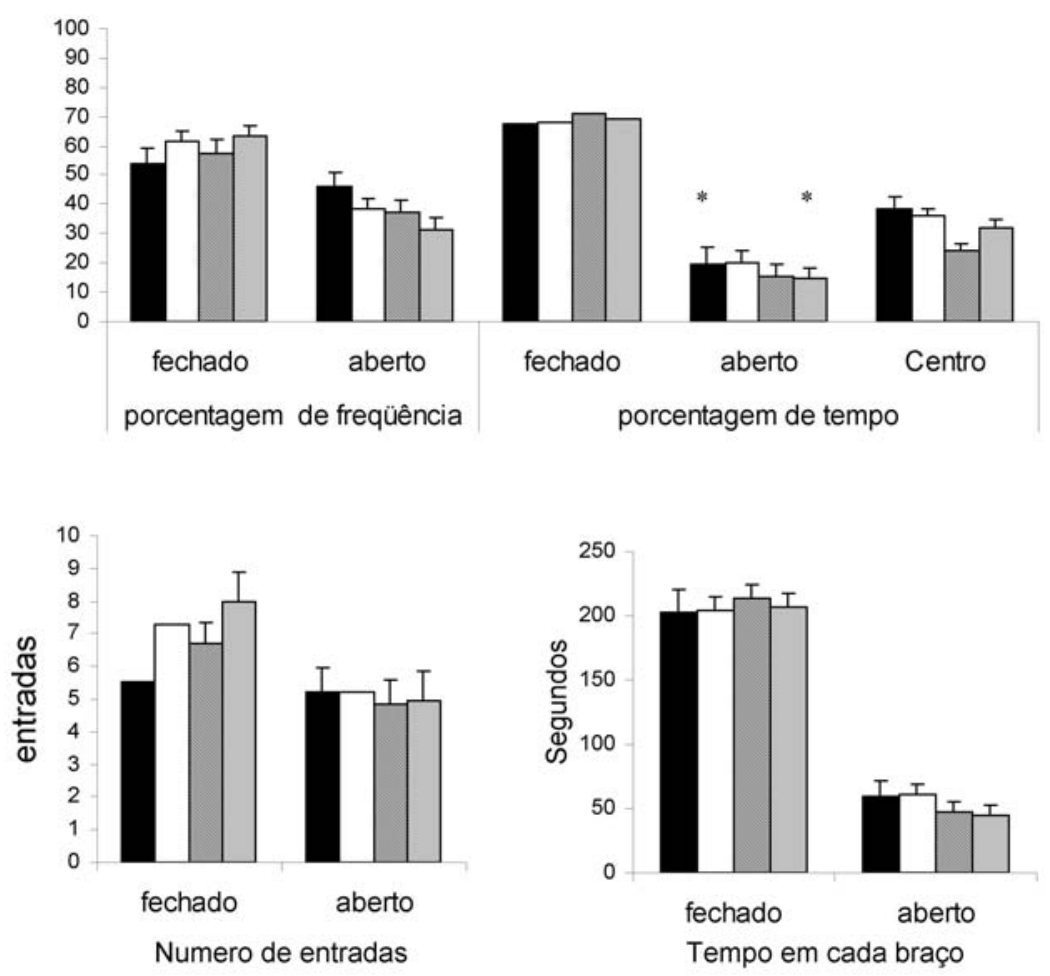

Figura 2 - Média \pm erro padrão dos resultados dos grupos experimentais. $\left(^{*}\right)$ indica diferença significativa entre os grupos $(P<0,001$, Teste de Tukey).

O número médio de entradas nos braços fechados foi maior no grupo em Diestro que nos demais estados hormonais embora a grande dispersão do grupo, evidenciada pelo erro padrão, tenha impedido que se constatasse diferenças estatísticas entre os grupos. O grupo de machos foi o que apresentou a freqüência média mais baixa de entradas nos braços fechados. Com relação ao número de entradas nos braços abertos, o Grupo Proestro apresentou a maior freqüência e o Grupo Estro, a menor.

Com relação ao tempo de permanência nos braços, os grupos Proestro e machos apresentaram um maior tempo de permanência nos braços abertos em detrimento da permanência nos braços fechados, que foi maior nos grupos Estro e Diestro. A porcentagem de entradas nos braços abertos foi maior nos grupos de machos e em Proestro, em comparação com os demais. Essas diferenças foram semelhantes quando se considerou o tempo gasto nos braços abertos.

A análise estatística indicou diferenças significativas na porcentagem de tempo nos braços abertos $(F[1,65]=47,213, P \leq 0,001)$, e o teste de Tukey indicou que a diferença se concentra entre os grupos de machos e Diestro. A porcentagem de entradas nos braços abertos apresentou tendência a diferenças, com resultados no limite da significância $(F[3,71]=2,679$, $\mathrm{P}=0,054)$. Em ambos os casos foi considerado o peso dos sujeitos como covariante.

Os dados foram interpretados com base nos critérios adotados, pelos quais a exploração dos braços abertos indicaria um índice de ansiedade, em contraste com o número de entradas nos braços fechados, que seria um índice de ambulação. Presume-se que o animal está submetido a dois drives contraditórios, um, de explorar um ambiente novo, outro, de proteger-se de uma possível predação, que se ampliaria pela exposição ao braço aberto e seria fonte da aversão incondicionada do animal por espaços abertos, também presente no Campo Aberto. Nesta perspectiva, a permanência no centro seria uma medida de conflito entre estes drives.

Os dados parecem indicar que a fase de Diestro favorece o aparecimento de maior ansiedade nos animais, indicada na menor porcentagem de entradas nos braços abertos, um índice amplamente aceito de aumento de ansiedade neste teste (Pellow e cols., 1985). É curioso notar que este aumento de ansiedade correlaciona-se a um aumento da ambulação (isto é, a quantidade total de deslocamento espacial do sujeito), indicado pela alta freqüência de entradas tanto nos braços abertos como nos fechados, em comparação 
com os outros grupos. Nesse sentido, é interessante notar que as fêmeas, nesta fase do ciclo, também parecem evitar expor-se aos braços abertos, caracterizando esquiva da estimulação aversiva gerada pelo espaço aberto. Este comportamento pode ser explicado pela ação ansiogênica da progesterona, cujo efeito deletério sobre a performance dos animais já foi demonstrado na literatura (Mora e cols., 1996; DiazVeliz e cols., 1997), e que é anulada pela presença do estradiol. Cabe lembrar (1) que o grupo Diestro do presente experimento incluiu animais de duas fases, o Metaestro e o Diestro propriamente dito, que não foram separados pela ausência de ação hormonal do folículo, e (2) que a ação da progesterona é tradicionalmente restrita ao primeiro dia de Proestro.

A menor freqüência de entradas dos machos em ambos os tipos de braços pode ser explicada pelo maior peso corporal dos animais em relação às fêmeas de todos os grupos. Isso, no entanto, não explica a semelhança entre este grupo e o grupo em Proestro no total de tempo despendido no centro, o qual teria melhor explicação pela ação conjunta do estrógeno e progesterona. O tempo no centro pode expressar um comportamento de conflito, que seria mais forte nos Machos que, poder-se-ia conjeturar, teriam maior nível de controle de impulsos.

A ausência de diferenças estatísticas nos outros índices, além da porcentagem de tempo, pode ser atribuída à grande dispersão encontrada nos dados, devida em parte à decisão experimental de considerar as fases de Metaestro e Diestro como uma só. Isto pode ter sido uma falha que deve ser considerada em outros experimentos que venham a tomar o ciclo estral como uma variável crítica.

A ambulação pode estar sob controle de outras variáveis aqui não manipuladas, como, por exemplo, a iluminação (Mora e cols., 1996).

Nossos dados replicaram os dados de Mora relativos à condição de baixa luz e relativos à porcentagem de entradas nos braços abertos mas não os dados relativos à porcentagem de tempo dispedido nestes braços. Tais diferenças podem se dever a diferenças no aparato (tamanho das paredes, de $20 \mathrm{~cm}$ no Trabalho de Mora e cols. (1996) e de $40 \mathrm{~cm}$ no nosso trabalho, por não havermos manipulado aqui as condições de luz ou ainda pelo fato de que a altura do Labirinto usado por Mora é de $70 \mathrm{~cm}$, contra de $50 \mathrm{~cm}$ em nosso trabalho).

Nossos dados apresentam uma alta dispersão, indicada pelos erros-padrão apresentados. No entanto, apesar de a dispersão de dados ser alta, ainda é menor do que aquela encontrada em estudos sobre diferenças sexuais, demonstrando que o ciclo estral influencia o grau de diferenciação entre gêneros no desempenho no Labirinto em cruz elevado.
Os dados aqui apresentados ampliam a nossa compreensão do efeito do ciclo hormonal sobre ansiedade e podem lançar luzes sobre os vários efeitos comportamentais dos hormônios. Também indicam a necessidade de mais estudos paramétricos para verificar quais elementos do aparato alteram os parâmetros de resposta obtidos.

\section{REFERÊNCIAS}

Alonso, S. J.; Castellano, M. A.; Afonso, D. \& Rodriguez, M. (1991). Sex differences in behavioral despair and open field. Physiology and Behavior, 59(2), 547-550.

Alves, C. R. R. \& Silva, M. T. A. (2002). Modelos animais de psicopatologia: Esquizofrenia. Em H. J. Guillardi; M. B. B. P. Madi, P. P. Queiroz, M. C. Scoz (Orgs.), Sobre comporamento e cognição (p. 49-60), v. 10. Santo André: ESETec.

Anderson, S. M.; Saviolaskis, G. A.; Bauman, R. A.; Chu, K. Y.; Ghosh, S. \& Kant, G. J. (1996). Effects of chronic stress on food acquisition, plasma hormones and the Estrous cycle of female rats. Physiology and Behavior, 60(1), 325-329.

Barros, H. T. M. \& Ferigolo, M. (1998). Ethopharmacology of imipramine in the forced swimming test: gender differences. Neurosciences and Biobehavioral Reviews, 23(2), 279-286.

Beatty, W. W. \& Beatty, P. A. (1970). Hormonal determinants sex differences in avoidance behavior and reactivity to electrical shocks in the rat. Journal of Comparative and Physiological Psychology, 73(3), 446-455.

Becker, A. \& Grecksch, G. (1996). Illumination has no effects on rats' behavior in the elevated plus maze. Physiology and Behavior, 59, 1175-1177.

Blanchard, R. J.; Franelly, K. J. \& Blanchard, C. D. (1986). Defensive behaviors of laboratory and wild Rattus Norvegicus. Journal of Comparative Psychology, 100(2), 101-107.

Breedlove, S. M. (1993). Sexual differentiation of the brain and behavior. Em J. B. Becker; S. M. Breedlove \& D. Crews (Orgs.), Behavioral Endocrinology (p. 39-70). $3^{\mathrm{a}}$ reimpressão. MIT Press, Cambridge, Mass.

Brown K. J. \& Grunberg, N. E. (1995). Effects of housing on male and female rats: Crowning stresses males but calm females. Physiology and Behavior, 58(6), 10851089.

Carter, C. S. (1993). Neuroendocrinology of sexual behavior in the female. Em J. B. Becker; S. M. Breedlove \& D. Crews (Orgs.), Behavioral Endocrinology (p. 71-96). $3^{\mathrm{a}}$ reimpressão. MIT Press, Cambridge, Mass.

Clarke, S. N. D. A. \& Ossenkopp, K. P. (1998). Taste reactivity responses in rats: influences of sex and the Estrous cycle. American Journal of Physiology - Regulatory Integrative and Comparative Physiology, 43(3), R718-R724.

Cole, B. J.; Hillman, M.; Seidelmann, D.; Klewer, M. \& Jones, G. H. (1995). Effects of benzodiazepines receptor partial inverse agonists in the elevated plus maze test of anxiety in the rat. Psychopharmacology, 121, 118-126.

Curzon, G.; Haaren, D. J. \& Kennett, G. A. (1990). Sex differences in animal model of depression. Em P. Spencer \& B. Leonard (Orgs.), Antidepressants: A thirty years on (p. 101-108). Londres, UK: CSN Publications.

Damiani K. \& Hunziker, M. H. L. (1991). Desamparo aprendido I: uma comparação entre machos e fêmeas. Resumos da XXI Reunião Anual de Psicologia da Socie- 
dade de Psicologia de Ribeirão Preto (Resumo 17). Ribeirão Preto: SBP.

Diaz-Veliz, G.; Alarcón, T.; Espinoza, C.; Dussaubat, N. \& Mora, S. (1997). Kentaserin and anxiety levels: influences of gender, estrous cycle, ovariectomy and ovarian hormones in female rats. Pharmacology, Biochemistry and Behavior, 58(3), 637-642.

Diaz-Veliz, G.; Soto, V.; Dussaubat, N. \& Mora, S. (1989). Influences of the Estrous cycle, ovariectomy and estradiol replacement upon the acquisition of conditioned avoidance responses in rats. Physiology and Behavior, 46, 397-401.

Fernadez-Trisac, J. L.; Brime, J. I.; López-Sela, P.; Sánchez-Garrido, L.; Costales, M.; Argüelles, J. \& Vijande, M. (1998). Effects of castration and gonadal hormones on insulin-induced drinking. Pharmacology, Biochemistry and Behavior, 59, 521-526.

Gouveia Jr., A. (2001). Efeitos da administração aguda de imipramina sobre o desamparo aprendido de ratos machos e fêmeas. Acta Comportamentalia, 9(1), 19-30.

Gouveia Jr., A. (2001). Influências do estado hormonal no desempenho de ratos nos testes do Labirinto em $T$ elevado e do nado forçado. Tese de Doutorado, IP/USP. São Paulo, SP.

Handley, S. L. \& Mithani, S. (1984). Effects of alphaadrenoceptor agonists in a maze-exploration model of "fear" - motivated behavior. Naunyn-Schmiedeberg's Archives of Pharmacology, 327, 1-5.

Heinsbroek, R. P. W.; Van Haaren, F. V.; Poll, N. E. V. \& Steenbergen, H. L. (1991). Sex differences in the behavioral consequences of inescapable footshocks depend on time since shocks. Physiology and Behavior, 49, 1257-1263.

Hogg, S. (1996). A review of the validity and variability of the elevated plus-maze as an animal model of anxiety. Pharmacology, Biochemistry and Behavior, 53, 271275.

Johnston, A. \& File, S. E. (1991). Sex differences in animal tests of anxiety. Physiology and Behavior, 49, 245-250.

Kandel, E. R.; Schwartz J. H. \& Jessell, T. M. (Orgs.) (1995). Essentials in neural sciences and Behavior. Londres, UK: Prentice Hall International, Inc.

Kellogg, C. K. \& Barrett, K. A. (1999). Reduced progesterone metabolites are not critical for plus-maze performance of lactating female rats. Pharmacology, Biochemistry and Behavior, 63(3), 441-448.

Kennett, G. A.; Chaouloff, F.; Marcou, M. \& Curzon, G. (1986). Female rats are morevulnerable than males in animal model of depression: The possible role of serotonin. Brain Research, 312, 416-421.

Koch, M. (1998). Sensorimotor gating changes across the Estrous cycle in female rats. Physiology and behavior, 64(5), 625-628

Laviano, A.; Meguid, M. M.; Gleason, J. R.; Yang, Z. J. \& Renvyle, T. (1996). Comparison of long-term feeding pattern between male and female Fisher 344 rats: influences of the Estrous cycle. American Journal of Physiology - Regulatory, Integrative and Comparative Physiology, 39(2), R413-R419.

Liversey, G. T.; Balabkins, N. \& Vogel, W.H. (1987). The effects of ethanol (alcohol) and stress on plasmacathecholamines levels in individual female an male rats. Neuropsychobiology, 17(4), 193-198.

Marvan, M. L.; Chavez-Chavez, L. \& Santana, S. (1986). Clomipramine modifies fluctuation $\mathrm{f}$ forced-swimming immobility in different phase of the rat estrous cycle. Archives of Medical Research, 27, 83-86.

Montgomery, K. C. (1955). The relationship between fear induced by novel stimulation and exploratory behavior. Journal of Comparative and Physiological Psychology. 48, 254-260.

Mora, S.; Dussaubat, N. \& Diaz-Veliz, G. (1996). Effects of the Estrous cycle and ovarian hormones on behavioral indices of anxiety in female rats. Psychoneuroendocrinology, 21(7), 609-620.

Moran, P. W. \& Lewis-Smith, M. (1979). Learned helplessness and response difficulty. Bulletin of Psychonomic Society, 13, 250-252

Morato, S. \& Brandão, M. S. (1997). Paradoxical increase of exploratory behavior in the elevated plus-maze by rats exposed to two kinds of aversive stimuli. Brazilian Journal of Medical and Biological Research, 30, 11131120.

Othoni, E. (2000). Etholog 2.2 - A tool for the transcription and timing of behavior observation sessions. Behavior Research, Methods, Instruments \& Computers, 32(3), 446-449.

Padovan, C. M.; Del-Bel, F. A. \& Guimarães, F. S. (1996). Pinealectomy attenuates the effect of restraint on plus maze exploration in rats. Brazilian Journal of Medical and Biological Research, 29, 1031-1034.

Parra, A.; Padilla, M.; Segovia, S. \& Guilamón, A. (1990). Diferencias en sexo en indefección aprendida en la rata. Revista de Psicologia General Y Aplicada, 43(1), 17-22.

Pellow, S.; Chopin, P.; File, S. E. \& Briley, M. (1985). Validation of open:closed arm entries in an elevated plus-maze as a measure of anxiety in the rat. Journal of Neurosciences Methods, 14, 149-167.

Pellow, S. \& File, S. E. (1986). Anxiolytic and anxiogenic drug effects on exploratory activity in elevated plusmaze: a novel test of anxiety in the rat. Pharmacology, Biochemistry and Behavior. 24, 525-529.

Reddy, D. S. \& Kulkarni, S. K. (1999). Sex and Estrous cycle-dependent changes in neurosteroid and benzodiazepine effects on food consumption and plus-maze learning behaviors in rats. Pharmacology, Biochemistry and Behavior. 62(1), 53-60.

Ryan, S. M. \& Maier, S. F. (1988). The Estrous cycle and Estrogen modulate stress induced analgesia. Behavioral Neurosciences, 102(3), 371-380.

Schwartz, N. B. (1969). A model for the regulation of ovulation in the rat. Em E. B. Astwood (Org.), Recent progress in hormones research - Proceeding of the 1968 Laurentian hormones Conference. Nova York, NJ: Academic Press, vol. 25.

Silva, M. R. P.; Bernadi, M. M.; Nasello, A. G. \& Felicio, L. (1997). Influences of lactation on the motor activity and elevate plus maze behavior. Brazilian Journal of Medical and Biological Research, 30, 241-244.

Steenbergen, H. L.; Heinsbroek, R. P. W.; Van Hesta, A. V. \& Poll, N. E. V. (1990). Sex-dependents effects of inescapable shock administration on shuttlebox-escape performance and elevated plus maze behavior. Physiology and Behavior, 48, 571-576.

Warren, S. G. \& Juraska, J. M. (1997). Spatial and non spatial learning across the rat Estrous cycle. Behavioral Neurosciences, 111(2), 259- 266.

Zukowska-Grojec, Z.; Shen, G. H.; Capraro, P. A. \& Vaz, C. A. (1991). Cardiovascular neuropeptide Y and adrenergic response in stress are sexually differentiated. Physiology and Behavior, 49, 771-777. 
Recebido: 09.07.2002

Revisado: 01.10.2002

\section{Notas:}

${ }^{1}$ A inibição pré-pulso ou modelo de resposta ao alarme acústico se refere ao efeito inibitório do reflexo de alarme pela apresentação de estímulo de intensidade mais fraca antes do estímulo que produz o alarme, sendo considerado um modelo animal de esquizofrenia (Alves e Silva, 2002).

${ }^{2} \mathrm{O}$ autor agradece ao Prof. Dr. Ari Fernando Maia pelo auxílio na gramática e estilo.

\section{Sobre os autores}

Amauri Gouveia Junior: Doutor em psicologia (Neurociências e comportamento), Universidade Estadual Paulista "Júlio de Mesquita Filho" - Departamento de Psicologia, Faculdade de Ciências, UNESP - Campus Bauru - Av. Luiz Edmundo C. Coube, S/N 17045-001 Bauru, SP-E-mail: agjunior@fc.unesp.br.

Silvio Morato: Livre-docente, Faculdade de Filosofia, Ciências e Letras de Ribeirão Preto - USP 